\title{
Substantial improvements in methyl ketone production in E. coli and insights on the pathway from in vitro studies
}

\author{
Ee-Been Goh $\uparrow^{1,3}$, Edward E. K. Baidoo ${ }^{1,3}$, Helcio Burd ${ }^{1,3}$, \\ Taek Soon Lee ${ }^{1,3}$, Jay D. Keasling ${ }^{1,3,4}$, Harry R. Beller* ${ }^{1,2}$ \\ ${ }^{1}$ Joint BioEnergy Institute, 5885 Hollis Avenue, Emeryville, CA 94608 \\ ${ }^{2}$ Earth Sciences Division, Lawrence Berkeley National Laboratory, Berkeley, CA 94720 \\ ${ }^{3}$ Physical Biosciences Division, Lawrence Berkeley National Laboratory, Berkeley, CA 94720 \\ ${ }^{4}$ Departments of Chemical \& Biomolecular Engineering and of Bioengineering, University of \\ California, Berkeley, CA 94720
}

\section{*Corresponding author:}

Harry R. Beller

Joint BioEnergy Institute

5885 Hollis Avenue

Emeryville, CA 94608

E-mail $\quad$ HRBeller@lbl.gov

Phone (510) 486-7321

Fax (510) 486-5686 


\section{Abstract}

3 We previously reported development of a metabolic pathway in E. coli for overproduction of

4 medium-chain methyl ketones (MK), which are relevant to the biofuel and flavor-and-fragrance

5 industries. This MK pathway was a re-engineered version of $\beta$-oxidation designed to

6 overproduce $\beta$-ketoacyl-CoAs and involved overexpression of the $f a d M$ thioesterase gene. Here,

7 we document metabolic engineering modifications that have led to a MK titer of $3.4 \mathrm{~g} / \mathrm{L}$ after

$8 \sim 45 \mathrm{hr}$ of fed-batch glucose fermentation and attainment of $40 \%$ of the maximum theoretical

9 yield (the best values reported to date for MK). Modifications included balancing

10 overexpression of $f a d R$ and $f a d D$ to increase fatty acid flux into the pathway, consolidation of the

11 pathway from two plasmids into one, codon optimization, and knocking out key acetate

12 production pathways. In vitro studies confirmed that a decarboxylase is not required to convert

$13 \beta$-keto acids into MK and that FadM is promiscuous and can hydrolyze several CoA-thioester

14 pathway intermediates.

15

\section{Keywords}

17 Methyl ketones; fatty acid; biofuels; FadM; beta-oxidation; acetate 


\section{Introduction}

Aliphatic methyl ketones are naturally occurring compounds (Forney and Markovetz,

21 1971) that can serve several different commercial applications. For example, medium-chain

22 methyl ketones such as 2-undecanone and 2-tridecanone are used in the flavor and fragrance

23 industry (Longo and Sanromán, 2006) and were recently reported as promising diesel fuel

24 blending agents (Goh et al., 2012). Goh and co-workers demonstrated the production of over 380

$25 \mathrm{mg} / \mathrm{L}$ of $\mathrm{C}_{11}$ to $\mathrm{C}_{15}$ methyl ketones from glucose $(0.2 \%$, in tryptic soy broth) in an engineered $E$.

26 coli DH1 strain, which represented a 700-fold increase in titer relative to a fatty acid-

27 overproducing strain (Goh et al., 2012). The major features of this E. coli strain were a re-

28 engineered $\beta$-oxidation pathway designed to overproduce $\beta$-ketoacyl-CoAs and overexpression

29 of the $f a d M$ gene encoding a native thioesterase. Subsequently, Park and co-workers (Park et al.,

30 2012) described engineering of an E. coli MG1655 strain that was able to produce $14 \mathrm{mg} / \mathrm{L}$ of

31 methyl ketones ( $2 \%$ glucose) when overexpressing a $\beta$-ketoacyl-ACP thioesterase (shmks 2$)$ and

32 a $\beta$-keto acid decarboxylase (shmks1) from Solanum habrochaites (wild tomato) and when adhE,

33 ldhA, poxB, and pta were deleted from the chromosome. Batch incubations of the best strain

34 under optimized growth conditions with 5\% glucose resulted in a methyl ketone titer of ca. 500

$35 \mathrm{mg} / \mathrm{L}$. In 2013, Müller et al. (2013) transferred the methyl ketone pathway of Goh and co-

36 workers into Ralstonia eutropha, a bacterium that can grow chemolithoautotrophically with $\mathrm{CO}_{2}$

37 and $\mathrm{H}_{2}$ as the sole carbon source and electron donor, respectively. R. eutropha was reported to

38 produce up to $180 \mathrm{mg} / \mathrm{L}$ of methyl ketones under such chemolithoautotrophic growth conditions

39 (Müller et al., 2013). Lan et al. (2013) produced 2-pentanone (for possible biomedical

40 application) in E. coli strain BW25113 by using a metabolic pathway that, unlike the other

41 studies cited above, did not rely on fatty acid precursors. More specifically, the pathway 
42 involved a biosynthetic thiolase reaction catalyzed by BktB to condense acetyl-CoA with

43 butyryl-CoA, forming $\beta$-ketohexanoyl-CoA, which was converted by a PcalJ-catalyzed CoA-

44 transferase reaction to $\beta$-ketohexanoic acid, which was then converted to 2-pentanone by

45 acetoacetate decarboxylase (Adc). The best reported 2-pentanone titer in that study was 240

$46 \mathrm{mg} / \mathrm{L}$ using $4 \%$ glucose in Terrific Broth (Lan et al., 2013). Another pathway for the synthesis of

47 short-chain methyl ketones was recently reported by Yoneda et al. (2014) for the production of

48 2-butanone in E. coli. The pathway builds on the 2,3-butanediol biosynthetic pathway and uses a

$49 \quad \mathrm{~B}_{12}$-dependent glycerol dehydratase from Klebsiella pneumoniae to convert 2,3-butanediol to 2-

50 butanone. Further co-overexpression of the $\operatorname{orf} z$ and $\operatorname{orf} 2 B$ genes, which encode the co-enzyme

$51 \quad \mathrm{~B}_{12}$ reactivator, resulted in a 2-butanone titer of $151 \mathrm{mg} / \mathrm{L}$ in minimal medium containing 3.6\%

52 glucose (Yoneda et al., 2014).

53 In this paper, we provide insights from further characterization of the methyl ketone

54 pathway first reported by Goh et al. (2012) using in vitro studies with purified pathway enzymes.

55 In addition, we detail the engineering of a series of pathway and host modifications that

56 improved the titer of the best methyl ketone-producing E. coli strain reported by Goh et al.

57 (2012) more than 160-fold in minimal medium to $1.4 \mathrm{~g} / \mathrm{L}$ (1\% glucose, shake flasks), which

58 represents $40 \%$ of the maximum theoretical yield. In addition, using fed-batch fermentation, we

59 were able to achieve a methyl ketone titer of $3.4 \mathrm{~g} / \mathrm{L}$ after $45 \mathrm{hr}$ of production.

\section{2. Materials and Methods}

61 2.1. Bacterial strains, plasmids, oligonucleotides, and reagents

62 Bacterial strains and plasmids used in this study are listed in Table 1. Strains and

63 plasmids along with their associated information (annotated GenBank-format sequence files)

64 have been deposited in the public version of the JBEI Registry (https://public-registry.jbei.org; 
65 entries JPUB_XXXX [to be added in proofs]) and are physically available from the authors

66 and/or addgene (http://www.addgene.org) upon request. Restriction enzymes were purchased

67 from Thermo Scientific (Waltman, MA), and Phusion DNA polymerase and T4 ligase were from

68 New England Biolabs (Ipswich, MA). Plasmid extractions were carried out using the QIAGEN

69 (Valencia, CA) miniprep kits. Oligonucleotide primers were designed using the web-based

70 PrimerBlast program (http://www.ncbi.nlm.nih.gov/tools/primer-

71 blast/index.cgi?LINK LOC=BlastHomeAd) and synthesized by Integrated DNA Technologies

72 (IDT), Inc (San Diego, CA) or Eurofins MWG Operon (Huntsville, AL). Primer sequences for

73 amplification of E. coli DH1 and Micrococcus luteus ORFs are listed in Table S1. The coding

74 sequences (CDS) of acyl-CoA oxidase (Mlut_11700, ACO) from M. luteus and FadD from E.

75 coli were synthesized and codon optimized for expression in E. coli by GenScript, Inc.

76 (Piscataway, NJ). Codon-optimized sequences are listed in Table S2.

\section{2.2. Media and bacterial growth}

78 E. coli was propagated aseptically as previously described (Sambrook et al., 1989). E.

79 coli DH10B cells were used for cloning and LB medium was used for routine growth and

80 propagation of strains. When required, antibiotics were added to the growth medium at the

81 following final concentrations: chloramphenicol, $25 \mu \mathrm{g} / \mathrm{mL}$; kanamycin, $50 \mu \mathrm{g} / \mathrm{mL}$. TSB

82 (containing $0.2 \%$ glucose) or M9-MOPS minimal medium [M9 medium supplemented with 75

$83 \mathrm{mM}$ MOPS, $2 \mathrm{mM} \mathrm{MgSO}_{4}, 1 \mathrm{mg} / \mathrm{L}$ thiamine, $10 \mathrm{nM} \mathrm{FeSO}_{4}, 0.1 \mathrm{mM} \mathrm{CaCl}_{2}, 56 \mathrm{mM} \mathrm{NH}_{4} \mathrm{Cl}_{2}$ and

84 micronutrients including $3 \mathrm{mM}\left(\mathrm{NH}_{4}\right)_{6} \mathrm{Mo}_{7} \mathrm{O}_{24}, 0.4 \mathrm{mM}$ boric acid, $30 \mathrm{mM} \mathrm{CoCl}_{2}, 15 \mathrm{mM}$

$85 \mathrm{CuSO}_{4}, 80 \mathrm{mM} \mathrm{MnCl}_{2}$, and $10 \mathrm{mM} \mathrm{ZnSO}_{4}$ ] (Zhang et al., 2012a) with $10 \mathrm{~g} / \mathrm{L}$ or $20 \mathrm{~g} / \mathrm{L}$ glucose

86 as the sole carbon source was used for production. For production in M9-MOPS minimal

87 medium, strains were first adapted in the medium. Briefly, cells were grown in LB overnight and 
88 diluted into M9-MOPS minimal medium at a starting $\mathrm{OD}_{600}$ (optical density at $600 \mathrm{~nm}$ ) of $\sim 0.1$.

89 Cultures were grown until an $\mathrm{OD}_{600}$ value of $\sim 1.0$ was reached ( 24 hours) and re-diluted into

90 fresh M9-MOPS medium as before. This was repeated two more times before the adapted

91 cultures were stored as frozen glycerol stocks at $-80^{\circ} \mathrm{C}$ until needed. When monitoring oxygen

$92\left(\mathrm{O}_{2}\right)$ consumption and carbon dioxide $\left(\mathrm{CO}_{2}\right)$ production during methyl ketone production, the

93 Respiration Activity MOnitoring System (RAMOS) system (Kuhner AG, Basel, Switzerland)

94 was used. The determination of the oxygen transfer rate (OTR) and carbon dioxide transfer rate

95 (CTR) were carried out as described by Anderlei et al. (2004).

96 2.3. Plasmid and strain construction for heterologous expression in E. coli

97 Cloning of M. luteus and E. coli genes into expression plasmids was carried out by PCR

98 amplification with appropriate primers (Table S1) as previously described (Goh et al., 2012).

99 Proper clone construction was confirmed by DNA sequencing, which was performed by

100 Quintara Biosciences (Albany, CA). When required, expression of heterologous genes in

101 constructs was confirmed by extraction of proteins, tryptic digestion, and analysis of the

102 resulting peptides by electrospray ionization liquid chromatography-tandem mass spectrometry

103 (LC/MS/MS)(QSTAR Elite Hybrid Quadrupole TOF, Applied Biosystems). In-frame

104 chromosomal deletion of E. coli genes was carried out using the method of Datsenko and co-

105 workers (Baba et al., 2006; Datsenko and Wanner, 2000).

106 2.4. In vitro assays with methyl ketone pathway enzymes

$107 \quad$ His $_{6}$-tagged acyl-CoA oxidase (ACO), His ${ }_{6}$-tagged FadB, and His 6 -tagged FadM were

108 purified as previously described (Beller et al., 2010). All in vitro assays were conducted with 0.1

109 M potassium phosphate buffer ( $\mathrm{pH}$ 7.5) containing $200 \mathrm{mg} / \mathrm{L} \mathrm{BSA}$ and $1 \mathrm{mM}$ DTT in 4-mL

110 screw-cap glass vials (with polytetrafluoroethylene liners). To generate enoyl-CoAs, a reaction 
111 containing $10 \mathrm{mM}$ myristoyl-CoA (Sigma), $500 \mu \mathrm{g}(0.21 \mathrm{mM})$ of His 6 -tagged acyl-CoA oxidase,

112 and $0.1 \mathrm{mM} \mathrm{FAD}$ to a final volume of $350 \mu \mathrm{L}$, was incubated on a stir plate at $30^{\circ} \mathrm{C}$ for $1.5 \mathrm{hr}$.

113 Five microliters of catalase (Sigma) were then added to the reaction and incubated as before for

114 another 30 min at $30^{\circ} \mathrm{C}$ to remove $\mathrm{H}_{2} \mathrm{O}_{2}$ generated by the acyl-CoA oxidase reaction. To proceed

115 toward the latter steps of the pathway, $50 \mu \mathrm{L}$ of the acyl-CoA oxidase reaction mixture was

116 added to a new 4-mL screw-cap glass vial containing (1) $25 \mu \mathrm{g}(0.28 \mathrm{mM})$ of His 6 -tagged FadM,

117 (2) $500 \mu \mathrm{g}(0.12 \mathrm{mM})$ of His $_{6}$-tagged FadB and $25 \mu \mathrm{g}(0.28 \mathrm{mM})$ of His ${ }_{6}$-tagged FadM, or (3)

$118500 \mu \mathrm{g}(0.12 \mathrm{mM})$ of His $_{6}$-tagged FadB, $25 \mu \mathrm{g}(0.28 \mathrm{mM})$ of His ${ }_{6}$-tagged FadM, and $6 \mathrm{mM}$

$119 \mathrm{NAD}^{+}$, in a final volume of $250 \mu \mathrm{L}$. In addition, a fourth reaction containing $2 \mathrm{mM}$ of myristoyl-

$120 \mathrm{CoA}$ and $25 \mu \mathrm{g}(0.28 \mathrm{mM})$ of $\mathrm{His}_{6}$-tagged FadM was carried out to investigate hydrolysis of

121 myristoyl-CoA by FadM. All four reactions were performed in duplicate. To stop the in vitro

122 reactions at specific time points, $100 \mu \mathrm{L}$ of reaction mixture was removed and added to a glass

123 vial containing an equal volume of methanol (LC-MS grade, Burdick and Jackson) and stored at

$124-20^{\circ} \mathrm{C}$ until LC-TOF analysis. Methyl ketones in the reactions were analyzed by LC-APCI-TOF

125 (liquid chromatography-atmospheric pressure chemical ionization-time of flight) mass

126 spectrometry, while all other metabolites were analyzed by LC-ESI (electrospray ionization)-

127 TOF mass spectrometry.

128 2.5. Analysis by LC-APCI-TOF and LC-ESI-TOF mass spectrometry

129 LC-APCI-TOF analyses were conducted at $55^{\circ} \mathrm{C}$ with a Kinetex XB-C18 column (100-

$130 \mathrm{~mm}$ length, 3-mm internal diameter, 2.6- $\mu \mathrm{m}$ particle size; Phenomenex, Inc., Torrance, CA)

131 using a 1200 Series HPLC (high-performance liquid chromatography) system (Agilent

132 Technologies, CA). The mobile phase was composed of water (solvent A) and methanol (solvent

133 B) (HPLC grade, Honeywell Burdick \& Jackson, CA). Two-microliter samples were injected 
134 and separated with the following gradient: $60 \%$ to $98 \%$ B for 3.47 min, held at $98 \%$ B for 5.2

$135 \mathrm{~min}$, decreased to $60 \% \mathrm{~B}$ for $2.42 \mathrm{~min}$, and held at $60 \% \mathrm{~B}$ for a further $2.78 \mathrm{~min}$. The total

136 analysis run time was $13.87 \mathrm{~min}$. A flow rate of $0.42 \mathrm{~mL} / \mathrm{min}$ was used throughout. APCI-TOF

137 MS conditions were described previously (Goh et al. 2012).

138 LC-ESI-TOF analyses were conducted at $55^{\circ} \mathrm{C}$ with the aforementioned Kinetex column

139 using a 1200 Series HPLC system (Agilent Technologies, CA). The mobile phase was composed

140 of $10 \mathrm{mM}$ ammonium acetate (pH 6.75) in water (solvent $\mathrm{A}$ ) and $10 \mathrm{mM}$ ammonium acetate in

141 methanol (solvent B) (HPLC grade, Honeywell Burdick \& Jackson, CA). Two-microliter

142 samples were injected and separated with the following gradient: $60 \%$ to $98 \%$ B for $3.47 \mathrm{~min}$,

143 held at $98 \%$ B for $5.2 \mathrm{~min}$, decreased to $60 \%$ B for $0.33 \mathrm{~min}$, and held at $60 \%$ B for a further 2

$144 \mathrm{~min}$. Metabolite separation was conducted via a flow rate of $0.42 \mathrm{~mL} / \mathrm{min}$ from 0 to $8.67 \mathrm{~min}$,

145 which was increased to $0.65 \mathrm{~mL} / \mathrm{min}$ from 8.67 to $9 \mathrm{~min}$ and held at this flow rate for an

146 additional $2 \mathrm{~min}$. The total analysis run time was $11 \mathrm{~min}$. The HPLC system was coupled to an

147 Agilent Technologies 6210 time-of-flight mass spectrometer (TOF MS) with a 1:5 post-column

148 split. Nitrogen gas was used as both the nebulizing and drying gas to facilitate the production of

149 gas-phase ions. The drying and nebulizing gases were set to $10 \mathrm{~L} / \mathrm{min}$ and $30 \mathrm{psi}$, respectively,

150 and a drying gas temperature of $325^{\circ} \mathrm{C}$ was used throughout. ESI was conducted in the negative-

151 ion mode with a capillary voltage of $3.5 \mathrm{kV}$. MS experiments were carried out in the full-scan

$152 \operatorname{mode}(\mathrm{m} / \mathrm{z} 170-1000)$ at 0.86 spectra per s for the detection of $[\mathrm{M}-\mathrm{H}]^{-}$ions. The instrument was

153 tuned for a range of $m / z 50-1700$. Prior to LC-ESI-TOF MS analysis, the TOF MS was

154 calibrated with the Agilent ESI-L TOF tuning mix.

155 Data acquisition and processing were carried out by the Agilent MassHunter Workstation

156 software. 2-Tridecanone, myristoyl-CoA, myristic acid, $\beta$-hydroxydecanoic acid (for 
157 quantification of $\beta$-hydroxytetradecanoic acid) and free CoA were available as authentic

158 standards and were quantified via an eight-point calibration curve ranging from $25 \mu \mathrm{M}$ to $1 \mathrm{mM}$.

159 Other analytes were identified by exact mass and their concentrations were estimated using the

160 most chemically similar compounds for which authentic standards were available.

161 2.6. Analyzing methyl ketones and fatty acids from cell cultures by gas chromatography-mass

162 spectrometry $(G C-M S)$

163 For methyl ketone production, frozen glycerol stocks of E. coli strains were used as

164 inocula and grown in $50 \mathrm{~mL}$ of TSB or M9-MOPS minimal medium (Zhang et al., 2012b) in

165250 -mL flasks with 200 -rpm agitation at $37^{\circ} \mathrm{C}$ for up to 96 hours. Final concentrations of 0.1 to

$1660.5 \mathrm{mM}$ of IPTG and $1 \mathrm{mM}$ of arabinose (unless indicated otherwise) were added to cultures

167 after 6 hours to induce gene expression. Five milliliters of decane (Sigma-Aldrich, ReagentPlus

$168 \geq 99 \%$ purity) or dodecane (for RAMOS experiments, Sigma-Aldrich, ReagentPlus $\geq 99 \%$

169 purity), amended with perdeuterated tetracosane $\left(\mathrm{C}_{24} \mathrm{D}_{50}\right)$ and 3-tetradecanone (Sigma-Aldrich)

170 as internal standards, were added to the cultures during induction. To measure methyl ketones

171 and fatty acids from cultures, $50 \mu \mathrm{L}$ of the decane overlay was removed at selected time points

172 for analysis by electron ionization (EI) GC-MS with a quadrupole mass spectrometer as

173 previously described (Goh et al., 2012).

174 2.7. Measuring glucose utilization and metabolite production during growth by high-

175 performance liquid chromatography (HPLC)

176 Glucose and organic acids from cell cultures were measured by an Agilent 1100 Series

177 HPLC system equipped with an Agilent 1200 Series refractive index detector (RID) (Agilent

178 Technologies, CA) and Aminex HPX-87H ion-exclusion column (300-mm length, 7.8-mm

179 internal diameter; Bio-Rad Laboratories, Inc., Hercules, CA). One hundred-microliter aliquots of 
180 cell cultures were removed at various time points during production and filtered through a spin-

181 cartridge with a $0.45-\mu \mathrm{m}$ nylon membrane, and $5 \mu \mathrm{L}$ of the filtrate was eluted through the

182 column at $50^{\circ} \mathrm{C}$ with $4 \mathrm{mM}$ sulfuric acid at a flow rate of $600 \mu \mathrm{L} / \mathrm{min}$ for $25 \mathrm{~min}$. Metabolites

183 were quantified by using external standard calibration with authentic standards.

184 2.8. Fed-batch fermentation for methyl ketone production

$185 \quad$ Fed-batch fermentation was carried out in a 2-L bioreactor equipped with a Sartorius

186 BIOSTAT ${ }^{\circledR} \mathrm{B}$ plus control unit for regulating dissolved oxygen (DO), $\mathrm{pH}$, and temperature. A

187 20-mL overnight culture prepared from a frozen glycerol stock was cultured in a 250-mL flask as

188 previously described. This culture was used to inoculate $710 \mathrm{~mL}$ of medium in the bioreactor.

189 The medium for batch phase was composed of M9 salts supplemented with $0.3 \mathrm{~g} / \mathrm{L}$ of

$190 \mathrm{MgSO}_{4} .7 \mathrm{H}_{2} \mathrm{O}, 5 \mathrm{~g} / \mathrm{L}$ of $\mathrm{NH}_{4} \mathrm{Cl}, 5 \mathrm{mg} / \mathrm{L}$ thiamine, $5.56 \mathrm{mg} / \mathrm{L}$ of $\mathrm{FeSO}_{4} .7 \mathrm{H}_{2} \mathrm{O}, 44 \mathrm{mg} / \mathrm{L}$

$191 \mathrm{CaCl}_{2} .2 \mathrm{H}_{2} \mathrm{O}$, micronutrients [consisting of $4 \mathrm{mM}\left(\mathrm{NH}_{4}\right)_{6} \mathrm{Mo}_{7} \mathrm{O}_{24}, 0.53 \mathrm{mM}$ boric acid, $40 \mathrm{mM}$

$192 \mathrm{CoCl}_{2}, 20 \mathrm{mM} \mathrm{CuSO}_{4}, 106 \mathrm{mM} \mathrm{MnCl}_{2}$, and $\left.13 \mathrm{mM} \mathrm{ZnSO}_{4}\right], 22 \mathrm{~g} / \mathrm{L}$ of glucose, and appropriate

193 antibiotics. One hundred and fifty milliliters of dodecane overlay amended with $0.5 \mathrm{~g} / \mathrm{L}$ of 3 -

194 tetradecanone as an internal standard was also added to the bioreactor at the beginning of batch

195 phase. The culture was maintained at $\mathrm{pH} 6.5$ automatically by the addition of a base solution (7

$196 \mathrm{M} \mathrm{NH}_{4} \mathrm{OH}$ and $5 \mathrm{M} \mathrm{KOH}$ ). The temperature was maintained at $37^{\circ} \mathrm{C}$ and airflow was sustained

197 at a rate of $0.1 \mathrm{VVM}$ (volume of air per volume of liquid per minute) throughout the

198 fermentation run. Dissolved oxygen was maintained at $22 \%$ via regulated stirrer speed. Cultures

199 were induced with $1 \mathrm{mM}$ arabinose and $0.1 \mathrm{mM}$ IPTG at 7 hours after initiation of batch phase.

200 Batch phase was carried out until glucose was depleted ( $22.8 \mathrm{hrs})$, at which time glucose

201 feeding was initiated. The feed solution contained approximately $505 \mathrm{~g} / \mathrm{L}$ glucose, $15 \mathrm{~g} / \mathrm{L}$

$202 \mathrm{MgSO}_{4} \cdot 7 \mathrm{H}_{2} \mathrm{O}$, micronutrients (at $3 \mathrm{X}$ concentration of the batch media) and appropriate 
203 antibiotics. The initial feed rate was set at $0.056 \mathrm{ml} / \mathrm{min}$. The feeding strategy employed an

204 automatic algorithm to create feed pulses by increasing the feed rate by $26 \%$ of the initial rate for

2052.5 minutes. Following this pulse, if the DO was more than $24 \%$, feed rate was increased by $7 \%$,

206 otherwise the new feed rate was decreased by 7\%. The feed rate was kept constant for 60 min

207 until the next pulse started. This cycle was repeated until all feed solution (ca. $577 \mathrm{~mL}$ ) had been

208 added to the fermenter.

209 3. Results and Discussion

210 3.1. Decarboxylation of $\beta$-keto acids to form methyl ketones occurs spontaneously

211 Unlike the pathway that we have developed, other recently reported heterologous

212 pathways for methyl ketone production include a decarboxylase to catalyze the final step,

213 namely, decarboxylation of a $\beta$-keto acid to form a methyl ketone (Park et al., 2012; Lan et al.,

214 2013). Previously, we hypothesized that the last (decarboxylation) step of our methyl ketone

215 pathway occurs spontaneously (Goh et al., 2012). This was supported by in vitro data, in which

216 assays containing only palmitoleyl-CoA [(Z)-9-hexadecenoyl-CoA], ACO, FadB, and required

217 cofactors were sufficient to generate a compound tentatively identified as (Z)-8-pentadecen-2-

218 one (15:1 methyl ketone) (Goh et al., 2012). To more definitively confirm our hypothesis, we

219 carried out in vitro assays with myristoyl-CoA (14:0), all three purified pathway enzymes, and

220 required cofactors and showed that, within an hour, the corresponding methyl ketone, 2-

221 tridecanone, was detected by LC-APCI-TOF (Figure 1A). The peak identified as 2- tridecanone

222 had the same retention time and mass-to-charge ratio $(\mathrm{m} / \mathrm{z})$ of the $[\mathrm{M}+\mathrm{H}]^{+}$ion (within $1.6 \mathrm{ppm}$ )

223 as an authentic 2-tridecanone standard. Furthermore, we observed near-quantitative conversion

224 of the $\mathrm{C}_{14} \beta$-keto acid to the $\mathrm{C}_{13}$ methyl ketone (2-tridecanone) (Figure 1B), demonstrating that

225 ACO, FadB, and FadM are sufficient to produce methyl ketones from acyl-CoAs without any 
226 decarboxylase; this assumes that ACO, FadB, and/or FadM do not have any unreported

227 decarboxylase activity. Although an authentic standard was not available for the $C_{14} \beta$-keto acid

228 (3-oxotetradecanoic acid), there were two key lines of evidence that strongly support its

229 identification. First, in the LC-APCI TOF data (Figure 1A), the putative $\mathrm{C}_{14} \beta$-keto acid peak

230 eluted much earlier than the $\mathrm{C}_{13}$ methyl ketone, as would be expected for a reverse-phase

231 separation of a carboxylic acid from a methyl ketone, and the $m / z$ ratio of its $[\mathrm{M}+\mathrm{H}]^{+}$ion was

232 virtually identical to that of the confirmed $\mathrm{C}_{13}$ methyl ketone (within $3.5 \mathrm{ppm}$ ). Thus, it appears

233 that the $C_{14} \beta$-keto acid was chromatographically separated from the $C_{13}$ methyl ketone but was

234 decarboxylated to form a methyl ketone when it entered the APCI ion source. Second, LC-ESI-

235 TOF analysis detected a peak with an $\mathrm{m} / \mathrm{z}$ value of 241.1826 for the $[\mathrm{M}-\mathrm{H}]^{-}$ion, which is within

$2366.6 \mathrm{ppm}$ of the expected $\mathrm{m} / \mathrm{z}$ value for a $\mathrm{C}_{14} \beta$-keto acid (Figure $\mathrm{S} 1$ ). The ESI ion source is lower

237 in energy than the APCI source and thus did not induce instantaneous decarboxylation.

238 3.2. In vitro assays show that the FadM thioesterase is promiscuous

239 In addition to confirming that decarboxylation of $\beta$-keto acids occurs spontaneously, in

240 vitro assays suggested that the FadM thioesterase also acts on CoA-thioester metabolites other

241 than $\beta$-ketoacyl-CoAs. This became apparent when free acid versions of the CoA-thioester

242 intermediates were detected when the methyl ketone pathway was allowed to progress to various

243 steps in the presence of FadM (the pathway is shown in Figure 2A). For example, incubation of

244 14:0 acyl-CoA (myristoyl-CoA) with FadM resulted in complete hydrolysis into myristic acid

245 within one hour (Figure 2B). In addition, 14:1 free acid was detected when ACO, FAD, and

246 FadM were present in the assay, although the rate of hydrolysis of the 14:1 acyl-CoA appears to

247 have been slower than that of the 14:0 acyl-CoA (i.e., the ratio of the 14:1 free acid to the CoA

248 thioester was relatively low after $1 \mathrm{hr}$ ) (Figure 2B). (When such experiments were conducted 
249 both in the presence and absence of FadM, the 14:1 free acid was only detected in the presence

250 of FadM, indicating that FadM was responsible for the hydrolysis (data not shown).) Further,

251 when myristoyl-CoA was incubated with ACO, FAD, FadB, and FadM to generate $\beta$ -

252 hydroxymyristoyl-CoA, there was complete hydrolysis to the $\beta$-hydroxy acid within $1 \mathrm{hr}$.

253 Finally, when myristoyl-CoA was incubated with ACO, FAD, FadB, NAD ${ }^{+}$, and FadM to

254 generate $\beta$-ketomyristoyl-CoA, only the $\beta$-keto acid and no $\beta$-ketoacyl-CoA was detected within

$2551 \mathrm{hr}$. The relatively broad substrate range of FadM is not surprising, as this enzyme was

256 previously shown to exhibit thioesterase activity toward a wide range of medium- and long-chain

257 CoA thioester intermediates from the $\beta$-oxidation pathway (Nie et al., 2008). However, the

258 relatively high apparent thioesterase activity of FadM on $\beta$-ketomyristoyl-CoA is interesting, and

259 this compound was not tested by Nie et al. (2008).

260 Our in vitro data suggest that tuning the expression of FadM relative to the rest of the

261 pathway enzymes may be important for maximizing flux to $\beta$-ketoacyl-CoAs by minimizing the

262 diversion of CoA-thioester intermediates to dead-end, free acid products. However, we should

263 point out that concentrations of these dead-end, free-acid products were negligible during in vivo

264 production (data not shown). Possible explanations for this observation are that: (1) in vitro and

265 in vivo conditions are different, including the absolute and relative concentrations of pathway

266 proteins, (2) the promiscuous activity of FadM could be masked in vivo by re-formation of CoA

267 thioesters catalyzed by FadD, and (3) flux through the methyl ketone pathway toward $\beta$ -

268 ketoacyl-CoAs may be sufficiently fast in vivo to preclude significant hydrolysis of CoA-

269 thioester intermediates by FadM.

270 3.3. Increasing fatty acid production and flux into the $\beta$-oxidation pathway for improved methyl

271 ketone production 
273 improvements to the pathway, including metabolically engineering strains to increase flux

274 through our modified version of $\beta$-oxidation. Methyl ketones derive from fatty acids (Figure 2A)

275 and conversion of fatty acids into acyl-CoAs, catalyzed by FadD (fatty acyl-CoA synthethase), is

276 the first committed step of $\beta$-oxidation and the gateway for entry of fatty acids into the methyl

277 ketone pathway (Figure 2A). In our previous report, free fatty acids were still detected at the end

278 of methyl ketone production by the best producing strain (EGS895), even though this strain

279 exhibited lower ratios of fatty acid precursors to their daughter methyl ketones compared to

280 lesser-producing strains (Goh et al., 2012). This suggested that there was suboptimal pull of free

281 fatty acids into the $\beta$-oxidation pathway. An initial attempt to increase the free fatty acid flux

282 into the methyl ketone pathway by overexpressing FadD was unsuccessful and resulted in a two-

283 fold decrease in methyl ketone production (Goh et al., 2012).

284 Another strategy for increasing methyl ketone production is to increase fatty acid

285 production by overexpressing the fatty acid-responsive transcription factor, FadR. It was

286 recently shown that overexpressing FadR increased free fatty acid titers 7.5 -fold in E. coli

287 (Zhang et al., 2012b). We evaluated this strategy to enhance methyl ketone production. The $f a d R$

288 gene was cloned in three different configurations to assess which would most enhance methyl

289 ketone titer: (1) $f a d R$ driven by the $\operatorname{trc}$ promoter ( $\left.\mathrm{P}_{\text {trc }}\right)$ (strain EGS1185; Table 1), (2) fadR cloned

290 downstream of $f a d M$ to create an operon driven by the $\mathrm{P}_{\text {trc }}$ promoter (strain EGS1190; Table 1),

291 and (3) fadR driven by the $\operatorname{araBAD}$ promoter ( $\mathrm{P}_{\mathrm{BAD}}$ ) (strain EGS1230; Table 1). Unfortunately,

292 all three configurations resulted in at least two-fold lower methyl ketone production than in the

293 absence of the cloned fadR gene, even though they increased fatty acid titer in some cases (e.g.,

294 strain EGS1230 had 1.6-fold higher free fatty acids than strain EGS895). We speculated that 
295 higher fatty acid titers were not translating into higher methyl ketone titers because conversion of

296 fatty acids to acyl-CoAs was a bottleneck. To test this, we co-overexpressed $f a d D$ with $f a d R$

297 (resulting in strain EGS1320; Table 1) and observed a ca. 4-fold increase in methyl ketone titer

298 (Figure 3). We also tested methyl ketone production by strain EGS1320 with varying inducer

299 (arabinose) concentrations to modulate $f a d R$ and $f a d D$ expression levels. At all tested

300 concentrations ( 0.5 to $5 \mathrm{mM}$ arabinose), methyl ketone production was always lower than in the

301 absence of arabinose, indicating that basal leaky expression of $f a d R$ and $f a d D$ was optimal for

302 methyl ketone production. Under these conditions, negligible amounts of free fatty acids were

303 detected in strain EGS1320 during production, suggesting that we had created a more efficient

304 metabolic pull of free fatty acids into the $\beta$-oxidation pathway (Figure S3).

305 We also attempted to use another strategy to increase the free fatty acid pool: enhancing

306 malonyl-CoA formation via acetyl-CoA carboxylase (ACC) overexpression. Davis et al. (2000)

307 observed a 6-fold enhancement in fatty acid titer in E. coli by overexpressing the native ACC

308 (consisting of four subunits: AccA, AccB, AccC, and AccD). However, they found that the

309 ratios of all four ACC subunits had to be balanced to avoid toxicity effects (Davis et al., 2000).

310 More recently, overexpression of the ACC complex from Corynebacterium glutamicum, which

311 consists of only two subunits (encoded by $a c c B C$ and $d t s R I$ ), was engineered into $E$. coli to

312 alleviate the bottleneck of malonyl-CoA for increased (2S)-flavanone production (Miyahisa et

313 al., 2005). Zha and co-workers subsequently confirmed that the overexpression of ACC from $C$.

314 glutamicum resulted in three-fold greater malonyl-CoA in E. coli (Zha et al., 2009). In light of

315 these data, we proceeded with overexpressing the ACC complex from C. glutamicum in our

316 engineered methyl ketone-producing strain (strain EGS1220, Table 1). Overexpressing the

317 heterologous ACC complex improved free fatty acid titers 4-fold but decreased methyl ketone 
318 production by almost ten-fold. We also cloned the ACC complex into strain EGS1320 (which

319 resulted in strain EGS1565, Table 1) but observed a two-fold decrease in methyl ketone

320 production relative to its parent strain. Since our attempts at overexpressing the ACC complex

321 tended to improve fatty acid titers but decrease methyl ketone titers, even in strains

322 overexpressing codon-optimized $f a d D$, we did not pursue this strategy further.

\section{3.4. Consolidating methyl ketone pathway enzymes onto one plasmid}

The methyl ketone pathway genes were originally borne on two plasmids (Figure 4A;

325 Table 1). We decided to consolidate the pathway genes onto a single plasmid so that one less

326 antibiotic would be needed to retain the plasmids through selection pressure. Our initial one-

327 plasmid strain, EGS1640, was obtained by sub-cloning the promoters and gene fragments from

328 both plasmids into the BglBrick vector, pBbE8k-RFP, which contained the colE1 origin of

329 replication (Lee et al., 2011). However, strain EGS1640 produced 14-fold lower methyl ketone

330 titers than the corresponding two-plasmid strain, EGS1370 (Figure S4). Targeted proteomics

331 analysis revealed that the acyl-CoA oxidase protein was very poorly expressed in EGS 1640.

332 Since the aco gene (Mlut_11700) was cloned from the Gram-positive actinobacterium,

333 Micrococcus luteus, whose genome has a GC content of $\sim 73 \%$ (Young et al., 2010), we codon-

334 optimized the gene for expression in E. coli. Replacing the original aco gene with a codon-

335 optimized version resulted in EGS1710 (Figure 4B), which not only had dramatically better

336 ACO expression than strain EGS1640 (Figure S4), but also had better ( 45\%) methyl ketone

337 production than strain EGS1370 (Figure 3).

338 After the pathway genes were reconfigured into a single plasmid for strain EGS1710, we

339 considered that the ratios of the expressed pathway enzymes may have changed and again tested

340 a range of inducer (IPTG and arabinose) concentrations. Indeed, we observed that optimal 
341 inducer concentrations had changed to $0.1 \mathrm{mM}$ IPTG and $1 \mathrm{mM}$ arabinose (recall that no

342 arabinose was the optimal condition for methyl ketone production in strain EGS1320).

343 3.5. Engineering of the E. coli host to reduce acetate production

344 Production of methyl ketones was initially performed in TSB complex medium (which

345 contains $0.2 \%$ glucose) (Goh et al. 2012). However, using complex or rich media such as TSB or

346 EZ-Rich would not be economically viable for large-scale production. The transition to use of

347 M9-MOPS minimal medium resulted in significant accumulation of acetate. Specifically, in 1\%

348 and $2 \%$ glucose, up to $2 \mathrm{~g} / \mathrm{L}$ and $6 \mathrm{~g} / \mathrm{L}$ of acetate was detected, respectively. Acetate

349 accumulation during growth is undesirable because it is known to inhibit protein expression even

350 at relatively low concentrations (e.g., $0.5 \mathrm{~g} / \mathrm{L}$ ) and also diverts carbon flux away from formation

351 of the desired products (Eiteman and Altman, 2006). Acetate production and excretion in E. coli

352 have been well characterized and occur via two major pathways under aerobic conditions

353 (Wolfe, 2005). In one pathway, pyruvate oxidase (PoxB) converts pyruvate to acetate, while in

354 another, phosphotransacetylase (Pta) converts acetyl-CoA to acetyl phosphate, and subsequently,

355 acetate kinase (AckA) dephosphorylates acetyl phosphate to acetate. We created chromosomal

356 knockouts of each of these pathways (ESG1710 with $\triangle$ poxB; EGS1890 with $\Delta a c k A-p t a$ ) as well

357 as the triple knockout (EGS1895 with $\triangle$ poxB and $\triangle a c k A-p t a$ ). Initially, we compared the two-

358 plasmid strain in either the pox $B^{+}$or poxB $^{-}$background (strain EGS1320 versus EGS1370,

359 respectively), and observed an 11-fold improvement in methyl ketone production in the pox $B^{-}$

360 strain compared to the pox $B^{+}$strain (Figure 3). Subsequently, we compared the one-plasmid

361 pathway in the various acetate-knockout hosts and observed that methyl ketone production in the

$362 \Delta a c k A-p t a$ background (strains EGS1890 and 1895) was about 2.5-fold greater than in the $\Delta$ poxB

363 background (strain EGS1710) (Figure 3). Measurement of acetate at the end of production 
364 showed none detectable in $1 \%$ glucose and $<3 \mathrm{~g} / \mathrm{L}$ in $2 \%$ glucose. Thus, knocking out the major

365 acetate pathways not only lowered acetate accumulation but also improved methyl ketone

366 production. The triple-knockout strain, EGS1895, is currently our best producer. In $1 \%$ glucose

367 (M9-MOPS minimal medium), it is capable of producing $\sim 1.4 \mathrm{~g} / \mathrm{L}$ of methyl ketones (Figure 3),

368 which represents $40 \%$ of the maximum theoretical yield.

369 3.6. Allocation of glucose carbon for the best methyl ketone producers

370 To better understand glucose metabolism in our three best methyl ketone-producing

371 strains (EGS1710, EGS1890, and EGS1895), we characterized glucose consumption and

372 production of methyl ketones, $\mathrm{CO}_{2}$, biomass, and acetate in these strains in the presence of $1 \%$

373 and $2 \%$ glucose over 90 hours (Figure 5). Noteworthy trends apparent in these data include the

374 following: (a) methyl ketone production was greater and glucose depletion more rapid in $\triangle a c k A$ -

$375 p t a$ knockout strains (EGS1890 and EG1895) compared to the $\triangle p o x B$ strain (EGS1710) and (b)

376 low levels of acetate (never exceeding $1 \mathrm{~g} / \mathrm{L}$ and $2 \mathrm{~g} / \mathrm{L}$ acetate for $1 \%$ and $2 \%$ glucose,

377 respectively) were released as glucose was consumed, and the acetate was re-assimilated

378 (presumably via AMP-forming acetyl-CoA synthetase (Wolfe, 2005)) upon complete depletion

379 of glucose. Interestingly, there was still acetate production in the triple acetate knockout strain

380 (especially with $2 \%$ glucose). In fact, acetate production was slightly higher in the $\Delta a c k A-p t a$

381 strains than in the $\Delta$ poxB strain (maximum of $\sim 1.7 \mathrm{~g} / \mathrm{L}$ versus $\sim 1.2 \mathrm{~g} / \mathrm{L}$, respectively), indicating

382 that acetate was being produced via an alternative pathway. One possible acetate source is from

383 the reaction of acetoacetyl-CoA transferase (encoded by atoA and atoD), whereby a fatty acid

384 and acetyl-CoA, which may be at higher concentrations in our engineered strains than for the

385 wild type, are converted to an acyl-CoA and acetate. The rate of acetate re-assimilation was also

386 different among the knockout strains. In the $\Delta p o x B$ strain (EGS1710), acetate was rapidly re- 
387 assimilated as soon as glucose consumption was complete. In contrast, in the $\triangle a c k A-p t a$ strains

388 (EGS1890 and EGS1895), acetate concentrations decreased slowly after glucose depletion but

389 acetate was never fully re-assimilated. This suggests that the $\triangle p o x B$ strain is better configured to

390 overcome the metabolic burden of assimilating acetate (1 ATP is required for the AMP-forming

391 acetyl-CoA synthetase) than the $\Delta a c k A-p t a$ strains. A potentially related observation is that the

$392 \Delta$ poxB strain (EGS1710) directed more glucose carbon to biomass after $90 \mathrm{hr}$ of incubation than

393 did the $\triangle a c k A-p t a$ strains (Table 2). Methyl ketone yields were generally comparable for strains

394 EGS1710, EGS1890, and EGS1895 in 1\% and 2\% glucose (Table 2), as were distributions of

395 individual methyl ketones (Table S4). Overall, loss of carbon to $\mathrm{CO}_{2}$ was substantial in all three

396 best strains (41-53\% of glucose carbon; Table 2). A relatively small portion of this $\mathrm{CO}_{2}$ loss can

397 be attributed to methyl ketone biosynthesis; based on the amount of methyl ketones produced

398 and accounting for decarboxylation reactions (pyruvate dehydrogenase, FabH and FabF, and

399 spontaneous $\beta$-keto acid decarboxylation) and carboxylation reactions (acetyl-CoA carboxylase)

400 in fatty acid and methyl ketone biosynthesis via our engineered pathway, approximately 3-8\% of

401 glucose carbon was released as $\mathrm{CO}_{2}$ attributable to our pathway. Thus, although we have

402 improved methyl ketone titers in minimal medium more than 160 -fold relative to our previously

403 reported best strain (Figure 3), there is still carbon flux to $\mathrm{CO}_{2}$ and biomass that could be more

404 efficiently diverted to methyl ketone production.

405 3.7. Fed-batch fermentation with best strain (EGS1895)

406 Fed-batch fermentation of strain EGS1895 demonstrated nearly linear methyl ketone

407 production over time while glucose was being fed, from approximately 23 to $45 \mathrm{hr}$, and methyl

408 ketone production corresponded well with glucose consumption (Figure 6). The maximum yield

409 during fermentation was $30 \%$ of the average value observed during batch production in shake 
410 flasks (0.042 g MK/g glucose, Figure 6, versus $0.14 \mathrm{~g}$ MK/g glucose, Figure 3). Acetate and

411 pyruvate concentrations remained relatively low in batch mode ( $2 \%$ glucose) but rose to 4.8 and

$4124.4 \mathrm{~g} / \mathrm{L}$, respectively, by $45 \mathrm{hr}$.

\section{4. Conclusions}

414 We have used in vitro studies to gain insights into the recently developed pathway (Goh

415 et al. 2012) that produces $\mathrm{C}_{11}$ to $\mathrm{C}_{17}$ methyl ketones in E. coli. In particular, these studies (1)

416 confirmed that a decarboxylase is not required to convert $\beta$-keto acids into methyl ketones (this

417 reaction occurs spontaneously) and (2) showed that FadM is promiscuous and can hydrolyze not

418 only $\beta$-ketoacyl-CoAs but also several CoA-thioester pathway intermediates, potentially

419 decreasing flux through the pathway by creating dead-end, free-acid products. However, these

420 dead-end products were not found to have accumulated in the growth medium during in vivo

421 production studies.

422 We have also made pathway and host engineering modifications to our best previously

423 reported methyl ketone-producing E. coli strain (Goh et al. 2012), resulting in a 160-fold titer

424 improvement in minimal medium. The best strain in the present study attained a methyl ketone

425 titer of $1.4 \mathrm{~g} / \mathrm{L}$ with $1 \%$ glucose in minimal medium, representing $40 \%$ of the maximum

426 theoretical yield. This strain reached a titer of $3.4 \mathrm{~g} / \mathrm{L}$ after $\sim 45 \mathrm{hr}$ of fed-batch fermentation

427 with glucose. Engineering improvements included increasing production of free fatty acids and

428 their flux into the methyl ketone pathway by balancing overexpression of the genes encoding the

429 fatty acid-responsive transcription factor, $f a d R$, and fatty acyl-CoA synthethase, $f a d D$. Multiple

430 parameters for $f a d R$ and $f a d D$ expression were tested individually and in combination $\left(\mathrm{P}_{\mathrm{BAD}}\right.$ and

$431 \mathrm{P}_{\text {trc }}$, a range of inducer concentrations, various operon configurations, and codon optimization for

$432 \mathrm{fad}(\mathrm{D})$, and the resulting methyl ketone titers were sensitive to these parameters. Attempts to 
433 increase fatty acid, and ultimately, methyl ketone production by overexpressing a heterologous

434 acetyl-CoA carboxylase resulted in increases in fatty acid production but sharp decreases in

435 methyl ketone production. Improvements in methyl ketone titer also resulted from consolidation

436 of the pathway on a single plasmid, codon optimization of the acyl-CoA oxidase from

437 Micrococcus luteus, and knockouts of key acetate production pathways (poxB, ackA, and pta).

438 Despite the large improvements in methyl ketone titer and yield, carbon mass balance studies

439 with 1 or $2 \%$ glucose demonstrated that there is still carbon flux to $\mathrm{CO}_{2}$ (41 to $53 \%$ of glucose

440 carbon) and biomass that could be more efficiently diverted to methyl ketone production. In the

441 future, we will leverage the use of metabolic flux analysis to determine which additional

442 modifications will most effectively improve our methyl ketone yields and titers.

\section{Acknowledgments}

444 We thank Pragya Singh and Christopher Petzold (Technology Division, JBEI) for mass

445 spectrometric analysis of protein samples. This work conducted by the Joint BioEnergy Institute

446 was supported by the Office of Science, Office of Biological and Environmental Research, of the

447 U.S. Department of Energy under Contract No. DE-AC02-05CH11231. E.-B.G. was partially

448 supported by Abengoa. J.D.K. has a financial interest in Amyris and Lygos.

449 Appendix A. Supplementary material

450 


\section{References}

452 Anderlei, T., Zang, W., Papaspyrou, M., Büchs, J., 2004. Online respiration activity

453 measurement (OTR, CTR, RQ) in shake flasks. Biochem. Eng. J. 17, 187-194. doi:10.1016/S1369-703X(03)00181-5

Baba, T., Ara, T., Hasegawa, M., Takai, Y., Okumura, Y., Baba, M., Datsenko, K.A., Tomita, M., Wanner, B.L., Mori, H., 2006. Construction of Escherichia coli K-12 in-frame, singlegene knockout mutants: the Keio collection. Mol. Syst. Biol. 2, 2006.0008. doi:msb4100050

Beller, H.R., Goh, E.B., Keasling, J.D., 2010. Genes involved in long-chain alkene biosynthesis in Micrococcus luteus. Appl. Environ. Microbiol. 76, 1212-1223. doi:AEM.02312-09 [pii] [pii] 10.1038/msb4100050

462 Datsenko, K.A., Wanner, B.L., 2000. One-step inactivation of chromosomal genes in 463 Escherichia coli K-12 using PCR products. Proc. Natl. Acad. Sci. U S A 97, 6640-6645.

464 Davis, M.S., Solbiati, J., Cronan Jr., J.E., 2000. Overproduction of acetyl-CoA carboxylase 465 activity increases the rate of fatty acid biosynthesis in Escherichia coli. J. Biol. Chem. 275, 466 28593-28598. doi:10.1074/jbc.M004756200 M004756200 [pii]

467 Eiteman, M.A., Altman, E., 2006. Overcoming acetate in Escherichia coli recombinant protein 468 fermentations. Trends Biotechnol. 24, 530-536. doi:S0167-7799(06)00215-0 [pii] $469 \quad$ 10.1016/j.tibtech.2006.09.001

470 Forney, F., Markovetz, A., 1971. The biology of methyl ketones. J. Lipid Res. 12, 383-395. 
471 Goh, E.B., Baidoo, E.E., Keasling, J.D., Beller, H.R., 2012. Engineering of bacterial methyl

472 ketone synthesis for biofuels. Appl. Env. Microbiol. 78, 70-80. doi:AEM.06785-11 [pii]

473 10.1128/AEM.06785-11

474 Kirchner, O., Tauch, A., 2003. Tools for genetic engineering in the amino acid-producing

475 bacterium Corynebacterium glutamicum. J. Biotechnol. 104, 287-299.

476 doi:S0168165603001482 [pii]

477 Lan, E.I., Dekishima, Y., Chuang, D.S., Liao, J.C., 2013. Metabolic engineering of 2-pentanone

$478 \quad$ synthesis in Escherichia coli. AIChE J. 59, 3167-3175. doi:10.1002/aic.14086

479 Lee, T.S., Krupa, R.A., Zhang, F., Hajimorad, M., Holtz, W.J., Prasad, N., Lee, S.K., Keasling,

480 J.D., 2011. BglBrick vectors and datasheets: A synthetic biology platform for gene

481 expression. J. Biol. Eng. 5. doi:1754-1611-5-12 [pii] 10.1186/1754-1611-5-12

482 Longo, M.A., Sanromán, M.A., 2006. Production of Food Aroma Compounds : Microbial and $483 \quad$ Enzymatic Methodologies. Food Technol. Biotechnol. 44, 335-353.

484 Meselson, M., Yuan, R., 1968. DNA restriction enzyme from E. coli. Nature 217, 1110-1114.

485 Miyahisa, I., Kaneko, M., Funa, N., Kawasaki, H., Kojima, H., Ohnishi, Y., Horinouchi, S., 486 2005. Efficient production of (2S)-flavanones by Escherichia coli containing an artificial 487 biosynthetic gene cluster. Appl. Microbiol. Biotechnol. 68, 498-504. doi:10.1007/s00253$488 \quad 005-1916-3$

489 Moran, U., Phillips, R., Milo, R., 2010. SnapShot: key numbers in biology. Cell 141, 1262 $490 \quad$ 1262.e1. doi:10.1016/j.cell.2010.06.019 
491 Müller, J., MacEachran, D., Burd, H., Sathitsuksanoh, N., Bi, C., Yeh, Y.-C., Lee, T.S., Hillson,

492 N.J., Chhabra, S.R., Singer, S.W., Beller, H.R., 2013. Engineering of Ralstonia eutropha

493 H16 for autotrophic and heterotrophic production of methyl ketones. Appl. Environ.

$494 \quad$ Microbiol. 79, 4433-9. doi:10.1128/AEM.00973-13

495 Nie, L., Ren, Y., Schulz, H., 2008. Identification and characterization of Escherichia coli

496 thioesterase III that functions in fatty acid $\beta$-oxidation. Biochemistry 47, 7744-7751.

497 doi:10.1021/bi800595f

498 Park, J., Rodríguez-Moyá, M., Li, M., Pichersky, E., San, K.-Y., Gonzalez, R., 2012. Synthesis

499 of methyl ketones by metabolically engineered Escherichia coli. J. Ind. Microbiol.

$500 \quad$ Biotechnol. 39, 1703-12. doi:10.1007/s10295-012-1178-x

501 Sambrook, J., Fritsch, E.F., Maniatis, T., 1989. Molecular cloning : a laboratory manual, 2nd ed.

502 Cold Spring Harbor Laboratory, Cold Spring Harbor, N.Y.

503 Steen, E.J., Kang, Y., Bokinsky, G., Hu, Z., Schirmer, A., McClure, A., Del Cardayre, S.B.,

504 Keasling, J.D., 2010. Microbial production of fatty-acid-derived fuels and chemicals from

505 plant biomass. Nature 463, 559-562. doi:nature08721 [pii] 10.1038/nature08721

506 Studier, F.W., Moffatt, B.A., 1986. Use of bacteriophage T7 RNA polymerase to direct selective

507 high-level expression of cloned genes. J. Mol. Biol. 189, 113-130. doi:0022-

$508 \quad 2836(86) 90385-2$ [pii]

509 Wolfe, A.J., 2005. The acetate switch. Microbiol. Mol. Biol. Rev. 69, 12-50. doi:69/1/12 [pii]

$510 \quad$ 10.1128/MMBR.69.1.12-50.2005 
511 Yoneda, H., Tantillo, D.J., Atsumi, S., 2014. Biological production of 2-butanone in Escherichia

$512 \quad$ coli. ChemSusChem 7, 92-5. doi:10.1002/cssc.201300853

513 Young, M., Artsatbanov, V., Beller, H.R., Chandra, G., Chater, K.F., Dover, L.G., Goh, E.-B.,

514 Kahan, T., Kaprelyants, A.S., Kyrpides, N., Lapidus, A., Lowry, S.R., Lykidis, A.,

515 Mahillon, J., Markowitz, V., Mavromatis, K., Mukamolova, G. V, Oren, A., Rokem, J.S.,

516 Smith, M.C.M., Young, D.I., Greenblatt, C.L., 2010. Genome sequence of the Fleming

$517 \quad$ strain of Micrococcus luteus, a simple free-living actinobacterium. J. Bacteriol. 192, 841-

518 860. doi:JB.01254-09 [pii] 10.1128/JB.01254-09

519 Zha, W., Rubin-Pitel, S.B., Shao, Z., Zhao, H., 2009. Improving cellular malonyl-CoA level in

520 Escherichia coli via metabolic engineering. Metab. Eng. 11, 192-198. doi:S1096-

$521 \quad 7176(09) 00012-3$ [pii] 10.1016/j.ymben.2009.01.005

522 Zhang, F., Carothers, J.M., Keasling, J.D., 2012a. Design of a dynamic sensor-regulator system

523 for production of chemicals and fuels derived from fatty acids. Nat. Biotechnol. 30, 354-9.

524 doi:10.1038/nbt.2149

525 Zhang, F., Ouellet, M., Batth, T.S., Adams, P.D., Petzold, C.J., Mukhopadhyay, A., Keasling,

526 J.D., 2012b. Enhancing fatty acid production by the expression of the regulatory

527 transcription factor FadR. Metab. Eng. 14, 653-60. doi:10.1016/j.ymben.2012.08.009 
530 Figure 1. Mass spectrometric data from in vitro assays demonstrating spontaneous (enzyme-

531 free) decarboxylation of $\beta$-keto acids to form methyl ketones. (A) LC-APCI-TOF

532 chromatogram of (top) in vitro assay initially containing tetradecanoyl-CoA, ACO, FadB, FadM,

$533 \mathrm{NAD}^{+}$, and FAD and incubated for $45 \mathrm{~min}$ and (bottom) 2-tridecanone authentic standard. (B)

534 Time course of in vitro assay showing concentrations of $\beta$-ketotetradecanoic acid (blue) and 2-

535 tridecanone (purple).

537 Figure 2. (A) Re-engineered $\beta$-oxidation pathway designed for methyl ketone production. Black 538 boxes represent overexpressed enzymes and gray circles represent genes that were

539 chromosomally deleted (see Goh et al., 2012 and text). The dashed arrows represent potential

540 side reactions catalyzed by the FadM thioesterase. (B) In vitro assay results showing

541 concentrations (determined by LC-ESI-TOF and LC-APCI-TOF mass spectrometry) of CoA-

542 thioester and $\beta$-keto acid intermediates, free acid dead-end products, and methyl ketones at $\mathrm{T}=0$

543 and 1 hour. Assay conditions allowing the reactions to progress through various pathway steps

544 are shown on the vertical axis. Approximately $1 \mathrm{mM}$ of myristoyl-CoA was added to the assays,

545 so it is clear that the detected metabolites typically accounted for more than $80 \mathrm{~mol} \%$ of this

546 substrate. A table containing the averages and standard deviations for the data represented in

547 Figure 2B are provided in Table S3. In addition, mass balance with respect to CoA (i.e., CoA-

548 thioester intermediates and free CoA) is presented in Figure S2.

550 Figure 3. History of strain configuration and corresponding titer in the development of a methyl

551 ketone pathway in E. coli based on re-engineered $\beta$-oxidation. Bar heights represent averages $(n$

$552 \geq 6$ ) and error bars represent 1 standard deviation of titers determined after $72 \mathrm{hr}$ of incubation;

553 total methyl ketone titers in $\mathrm{mg} / \mathrm{L}$ are indicated above the bars. "co- $a c o "$ represents a codon-

554 optimized version of acyl-CoA oxidase from Micrococcus luteus. Gray bars represent titers for

555 strains grown in TSB (0.2\% glucose) and reported by Goh et al. (2012) and black bars represent

556 strains grown in M9-MOPS minimal medium (1\% glucose). 
558 Figure 4. Plasmid maps of the (A) two-plasmid system used in strains EGS1320 and EGS1370

559 and (B) the one-plasmid system used in strains EGS1710, EGS1890, and EGS1895.

560

561 Figure 5. Time-course data for the three best producing strains in this study: EGS1710 ( $\triangle$ poxB),

562 EGS1890 ( $\triangle a c k A-p t a)$, and EGS1895 ( $\triangle a c k A-p t a, \Delta p o x B)$. Symbols: methyl ketones (red

563 triangles), glucose (blue diamonds), acetate (green squares), $\mathrm{OD}_{600}$ (black circles), $1 \%$ glucose

564 (solid lines with filled symbols), and $2 \%$ glucose (dotted lines with open symbols). Analytes not

565 detected included lactate, pyruvate, formate, and ethanol.

566

567 Figure 6. Fed-batch fermentation of strain EGS1895 with glucose. The initial batch phase with $5682 \%$ glucose ended at $\sim 23 \mathrm{hr}$, at which time glucose feeding commenced.

569

570 
571 TABLE 1. Bacterial strains and plasmids used in this study

\begin{tabular}{|c|c|c|}
\hline Strain or plasmid & Relevant Characteristics & Source or reference \\
\hline \multicolumn{3}{|l|}{ E. coli strains } \\
\hline BL21 (DE3) & $\mathrm{F}^{-}$ompT gal dcm lon hsdSB($\left(\mathrm{r}_{\mathrm{B}}^{-} \mathrm{m}_{\mathrm{B}}^{-}\right) \lambda(\mathrm{DE} 3)$ & $\begin{array}{l}\text { (Studier and } \\
\text { Moffatt. 1986) }\end{array}$ \\
\hline DH1 & $\begin{array}{l}\text { endAl recAl gyrA96 thi-1 glnV44 relAl hsdR17( } \mathrm{r}_{\mathrm{K}}^{-} \\
\left.\mathrm{m}_{\mathrm{K}}^{+}\right) \lambda^{-}\end{array}$ & $\begin{array}{l}\text { (Meselson and } \\
\text { Yuan, 1968) }\end{array}$ \\
\hline LT- $\square$ fadE & DH1 $\triangle f a d E$ with pKS1 & (Steen et al., 2010) \\
\hline EGS084 & LT- $\square$ fadE with pEC-XK99E & (Beller et al., 2010) \\
\hline EGS514 & BL21(DE3) with pEG513 & (Goh et al. 2012) \\
\hline EGS517 & BL21(DE3) with pEG516 & (Goh et al. 2012) \\
\hline EGS522 & $\mathrm{DH} 1 ; \Delta f a d E ; \Delta f a d A$ & (Goh et al. 2012) \\
\hline EGS560 & EGS522 with pEG530 and pEC-XK99E & (Goh et al. 2012) \\
\hline EGS860 & LT- $\Delta$ fadE with pEG855 & (Goh et al. 2012) \\
\hline EGS895 & EGS522 with pEG530 and pEG855 & (Goh et al. 2012) \\
\hline EGS1127 & BL21(DE3) with pEG1125 & This study \\
\hline EGS1185 & EGS522 with pEG530 and pEG1165 & This study \\
\hline EGS1190 & EGS522 with pEG530 and pEG1170 & This study \\
\hline EGS1220 & EGS522 with pEG530 and pEG1200 & This study \\
\hline EGS1230 & EGS522 with pEG530 and pEG1210 & This study \\
\hline EGS1320 & EGS522 with pEG530 and pEG1315 & This study \\
\hline EGS1350 & DH1; $\triangle f a d E ; \triangle f a d A ; \Delta p o x B$ & This study \\
\hline EGS1370 & EGS1350 with pEG530 and pEG1315 & This study \\
\hline EGS1400 & DH1; $\Delta f a d E ; \Delta f a d A ; \Delta a c k A-p t a$ & This study \\
\hline EGS1405 & DH1; $\triangle$ fadE; $\triangle$ fadA; $\triangle a c k A-p t a ; \Delta p o x B$ & This study \\
\hline EGS1565 & EGS522 with pEG530 and pEG1545 & This study \\
\hline EGS1640 & EGS1350 with pEG1610 & This study \\
\hline EGS1710 & EGS1350 with pEG1675 & This study \\
\hline EGS1890 & EGS1400 with pEG1675 & This study \\
\hline EGS1895 & EGS1405 with pEG1675 & This study \\
\hline \multicolumn{3}{|l|}{ Plasmids } \\
\hline pBbA5c-RFP & $\begin{array}{l}\mathrm{Cm}^{\mathrm{r}} \text {; p15a derivative BglBrick vector containing RFP } \\
\text { under the lac } \mathrm{UV} 5 \text { promoter }\left(\mathrm{P}_{\text {lacUv5 }}\right) \text {. }\end{array}$ & (Lee et al., 2011) \\
\hline pBbE8k-RFP & $\begin{array}{l}\mathrm{Km}^{\mathrm{r}} \text {; ColE1 derivative BglBrick vector containing } \\
\mathrm{RFP} \text { under the } a r a B A D \text { promoter }\left(\mathrm{P}_{\mathrm{BAD}}\right) \text {. }\end{array}$ & (Lee et al., 2011) \\
\hline pE8a-fadR & $\begin{array}{l}\text { Amp } \text {; ColE1 derivative containing } f a d R \text { under the } \\
\text { araBAD promoter }\left(\mathrm{P}_{\mathrm{BAD}}\right) \text {. }\end{array}$ & $\begin{array}{l}\text { (Zhang et al., } \\
\text { 2012b) }\end{array}$ \\
\hline pEC-XK99E & $\begin{array}{l}\mathrm{Km}^{\mathrm{r}} ; E \text {. coli }-C \text {. glutamicum shuttle expression } \\
\text { vectors based on the medium copy number plasmid } \\
\text { pGA1 and containing the } \operatorname{trc} \text { promoter }\left(\mathrm{P}_{\text {trc }}\right)\end{array}$ & $\begin{array}{l}\text { (Kirchner and } \\
\text { Tauch, 2003) }\end{array}$ \\
\hline pKS1 & $\mathrm{Cm}^{\mathrm{r}}$; $\mathrm{p} 15$ a derivative containing 'tesA under $\mathrm{P}_{\text {lacuv5 }}$ & (Steen et al., 2010) \\
\hline pEG513 & $\begin{array}{l}\mathrm{Km}^{\mathrm{r}} ; \sim 2.2-\mathrm{kb} \text { PCR fragment of } f a d B \text { cloned into } \\
\text { pSKB3 at } N d e \text { I and SalI sites. }\end{array}$ & (Goh et al. 2012) \\
\hline pEG516 & $\mathrm{Km}^{\mathrm{r}} ; \sim 2.1-\mathrm{kb}$ fragment of Mlut_11700 cloned into & (Goh et al. 2012) \\
\hline
\end{tabular}




\begin{tabular}{|c|c|c|}
\hline pEG530 & $\begin{array}{l}\text { pSKB3 at } N d e \text { I and SalI sites. } \\
\text { Cm'; } \sim 2.1-\mathrm{kb} \text { PCR fragment of Mlut_11700 and } \sim 2.2- \\
\mathrm{kb} \text { PCR fragment of } \operatorname{fadB} \text { cloned downstream of the } \\
\text { 'tesA gene in pKS1 by SLIC. }\end{array}$ & (Goh et al. 2012) \\
\hline pEG855 & $\begin{array}{l}\mathrm{Km}^{\mathrm{r}} ; \sim 0.4-\mathrm{kb} \text { PCR fragment of fadM cloned into pEC- } \\
\mathrm{XK} 99 \mathrm{E} \text { at EcoRI and XbaI sites. }\end{array}$ & (Goh et al. 2012) \\
\hline pEG1125 & $\begin{array}{l}\mathrm{Km}^{\mathrm{r}} ; \sim 0.4-\mathrm{kb} \text { PCR fragment of } f a d M \text { cloned into } \\
\text { pSKB3 at } N d e \text { I and SalI sites. }\end{array}$ & This study \\
\hline pEG1165 & $\begin{array}{l}\mathrm{Km}^{\mathrm{r}} ; \sim 0.25-\mathrm{kb} \text { PCR fragment of } \mathrm{P}_{\mathrm{trc}} \text { and } \sim 0.72-\mathrm{kb} \\
\text { PCR fragment of } \text { fadR cloned into SalI site of pEG855 } \\
\text { by SLIC. }\end{array}$ & This study \\
\hline pEG1170 & $\begin{array}{l}\mathrm{Km}^{\mathrm{r}} ; \sim 0.72-\mathrm{kb} \text { PCR fragment of } f a d R \text { cloned into SalI } \\
\text { site downstream of } f a d M \text { in pEG } 855 \text { by SLIC. }\end{array}$ & This study \\
\hline pEG1200 & $\begin{array}{l}\mathrm{Km}^{\mathrm{r}} ; \sim 2.0-\mathrm{kb} \text { PCR fragment of } \mathrm{P}_{\mathrm{trc}}-a c c B C \text { and } \sim 1.9- \\
\mathrm{kb} \text { PCR fragment of } \mathrm{P}_{\mathrm{trc}}-d t s R 1 \text { cloned into } S a l \mathrm{I} / \mathrm{Xba \textrm {I }} \\
\text { sites of pEG855 by SLIC. }\end{array}$ & This study \\
\hline pEG1210 & $\begin{array}{l}\mathrm{Km}^{\mathrm{r}} ; \sim 2.2-\mathrm{kb} \text { PCR fragment of } \operatorname{araC}-\mathrm{P}_{\mathrm{BAD}}-f a d R \text { of } \\
\text { pE8a-fadR cloned into NdeI site of pEG855 by SLIC. }\end{array}$ & This study \\
\hline pEG1315 & $\begin{array}{l}\mathrm{Km}^{\mathrm{r}} ; \sim 1.7-\mathrm{kb} \text { PCR fragment of codon optimized fadD } \\
\text { (see Table S2) cloned into BamHI site of pEG1210 by } \\
\text { SLIC. }\end{array}$ & This study \\
\hline pEG1545 & $\begin{array}{l}\mathrm{Km}^{\mathrm{r}} ; \sim 4.0-\mathrm{kb} \text { PCR fragment of } \mathrm{P}_{\text {trc }}-a c c B C-\mathrm{P}_{\text {trc }}-d t s R l \\
\text { from pEG1200 cloned into XbaI site of } \mathrm{pEG} 1315 \text { by } \\
\text { SLIC. }\end{array}$ & This study \\
\hline pEG1610 & $\begin{array}{l}\mathrm{Km}^{\mathrm{r}}, \sim 6.6-\mathrm{kb} \text { fragment of } \operatorname{araC}-\mathrm{P}_{\mathrm{BAD}}-f a d R-\mathrm{co} f a d D- \\
\mathrm{P}_{\mathrm{trc}}-f a d M \text { fragment from } \mathrm{pEG} 1315 \text { and } \sim 5.1-\mathrm{kb} \\
\text { fragment of } \mathrm{P}_{\text {lacUV5- }} \text { 'tes } A-f a d B-a c o \text { from } \mathrm{pEG530} \\
\text { cloned into pBbE8k-RFP at the BamHI and AatII sites. }\end{array}$ & This study \\
\hline pEG1615 & $\begin{array}{l}\mathrm{Cm}^{\mathrm{r}} \text {, same as pEG530 except Mlut_11700 was } \\
\text { replaced with a version that is codon-optimized for } \\
\text { expression in E. coli (see Table S2) using BamHI } \\
\text { sites. }\end{array}$ & This study \\
\hline pEG1675 & $\begin{array}{l}\mathrm{Km}^{\mathrm{r}}, \sim 6.6-\mathrm{kb} \text { fragment of } a r a C-\mathrm{P}_{\mathrm{BAD}}-f a d R-\mathrm{co} f \text { fadD- } \\
\mathrm{P}_{\text {trc }} \text {-fadM fragment from pEG1315 and } \sim 5.1-\mathrm{kb} \\
\text { fragment of } \mathrm{P}_{\text {lacUv5- }} \text { 'tesA-fadB-co_aco from pEG1615 } \\
\text { cloned into pBbE8k-RFP at the BamHI and AatII sites. }\end{array}$ & This study \\
\hline pSKB3 & $\begin{array}{l}\mathrm{Km}^{\mathrm{r}} \text {; A derivative of the expression vector } \mathrm{pET}-28 \mathrm{a} \\
\text { with the thrombin protease site replaced by a TEV } \\
\text { protease site. }\end{array}$ & Burleyc \\
\hline
\end{tabular}


Table 2. Carbon mass balance in three best methyl ketone-producing strains ${ }^{\mathrm{a}}$

\begin{tabular}{|c|c|c|c|c|c|c|}
\hline & Strains & $\begin{array}{l}\text { Methyl } \\
\text { ketones }^{b}\end{array}$ & Biomass $^{\mathrm{b}, \mathrm{c}}$ & $\mathrm{CO}_{2}{ }^{\mathrm{b}}$ & Acetate $^{b}$ & Total $^{b}$ \\
\hline \multirow{3}{*}{ 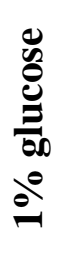 } & EGS1710 & 7.6 & 41 & 42 & 0.0 & 90 \\
\hline & EGS1890 & 17 & 27 & 53 & 0.0 & 97 \\
\hline & EGS1895 & 19 & 25 & 52 & 0.0 & 95 \\
\hline \multirow{3}{*}{ 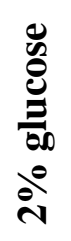 } & EGS1710 & 9.4 & 35 & 41 & 0.0 & 86 \\
\hline & EGS1890 & 14 & 26 & 42 & 4.0 & 86 \\
\hline & EGS1895 & 16 & 24 & 47 & 3.0 & 90 \\
\hline
\end{tabular}

${ }^{\mathrm{a}}$ Data are from the same experiment represented in Figure 5.

${ }^{\mathrm{b}}$ Expressed as \% of glucose carbon.

${ }^{\mathrm{c}}$ Assuming that $50 \%$ of cell dry weight is carbon (Moran et al., 2010) 
Figure 1
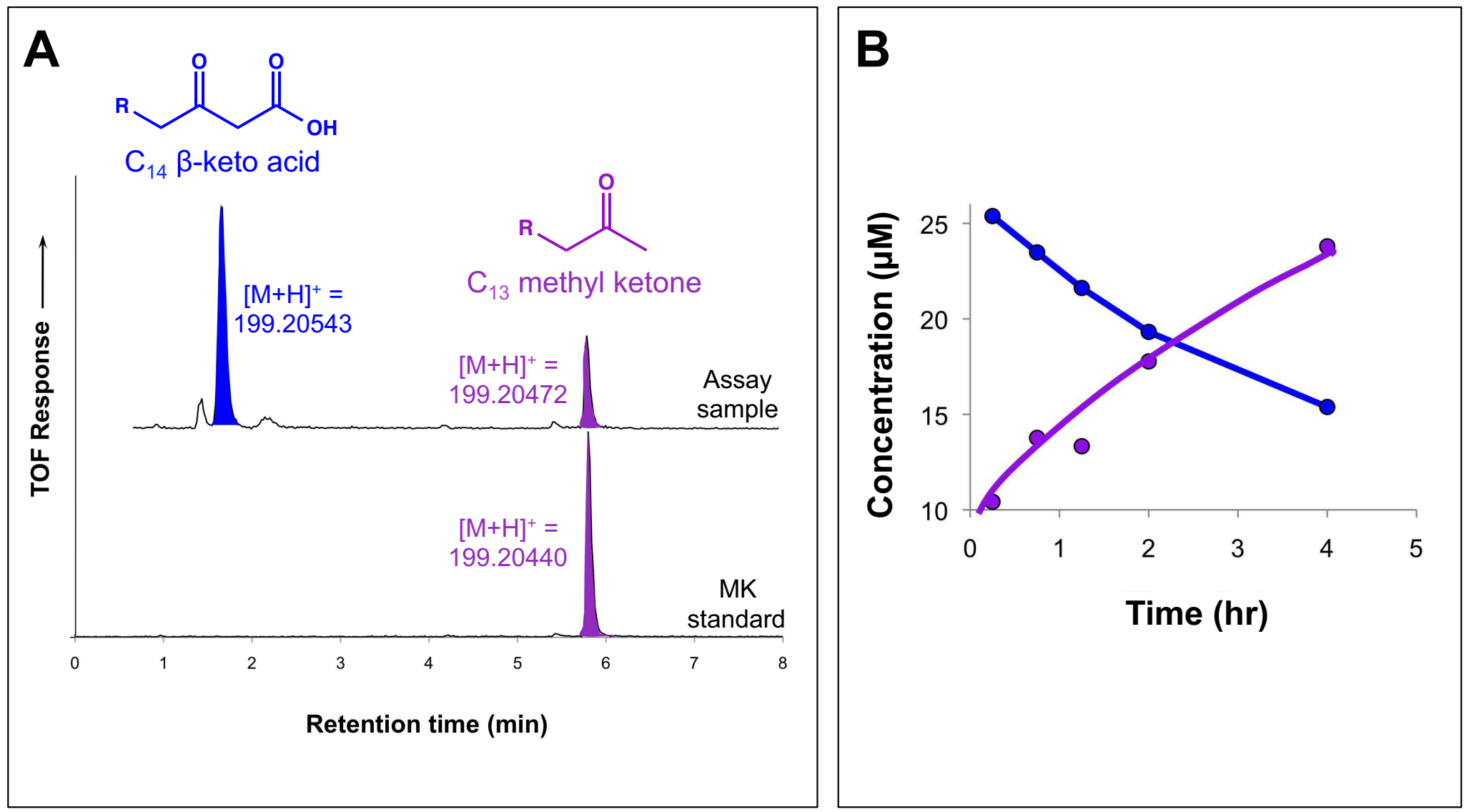
Figure 2

A

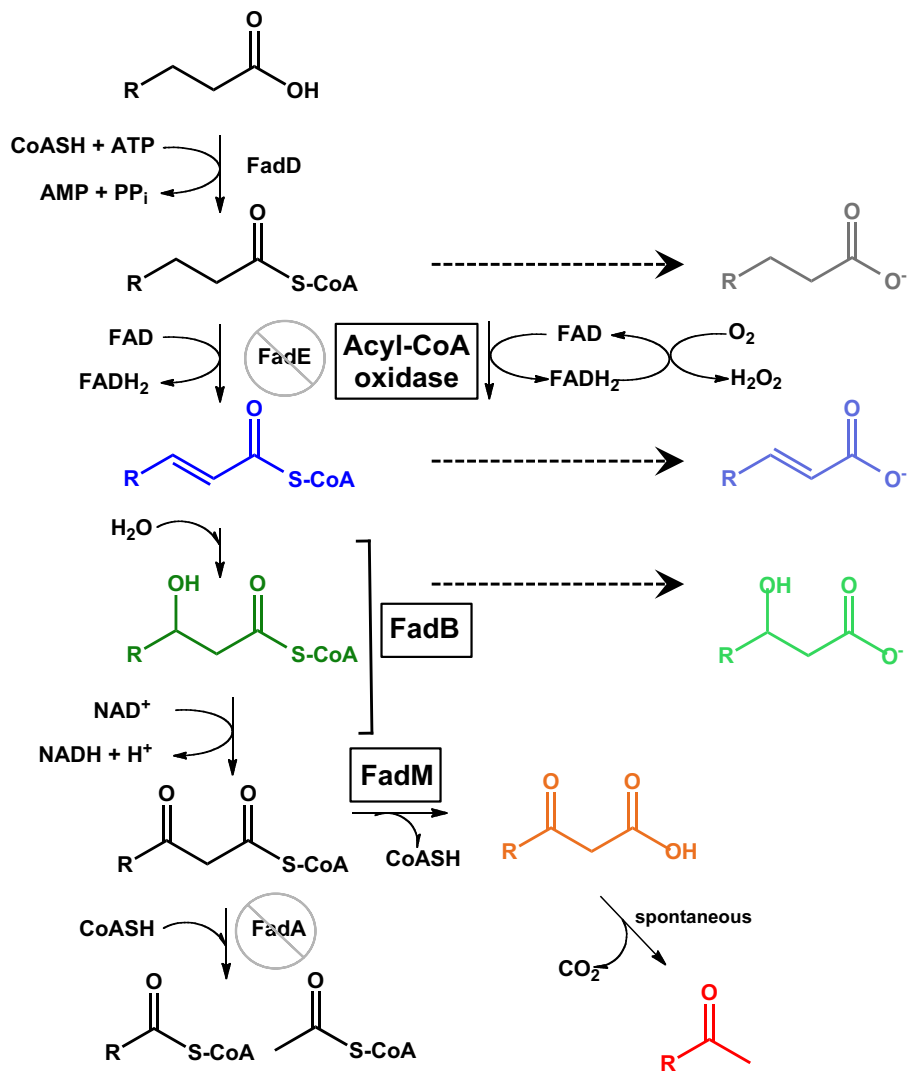

B

Concentration $(\mu \mathrm{M})$

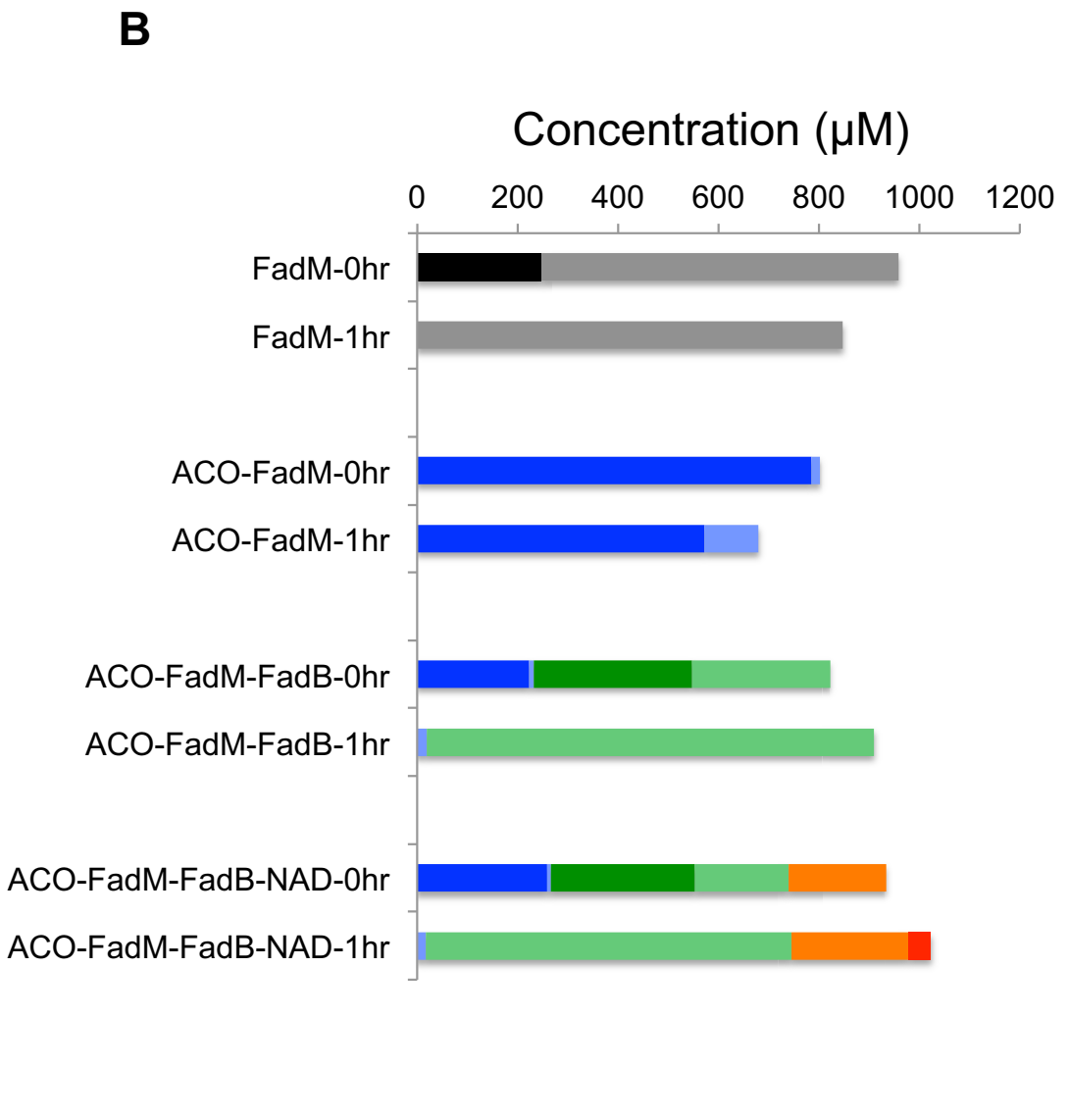




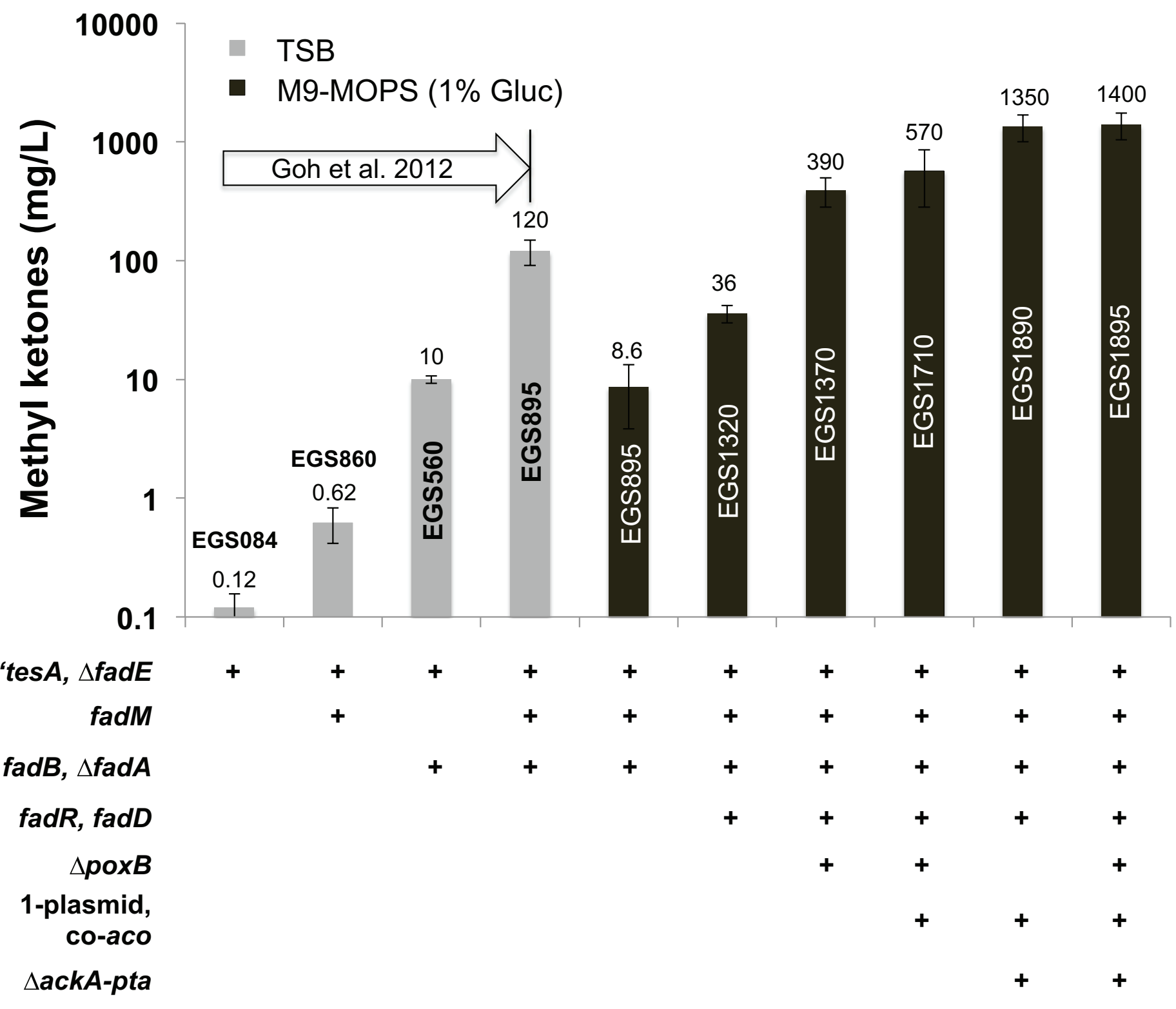



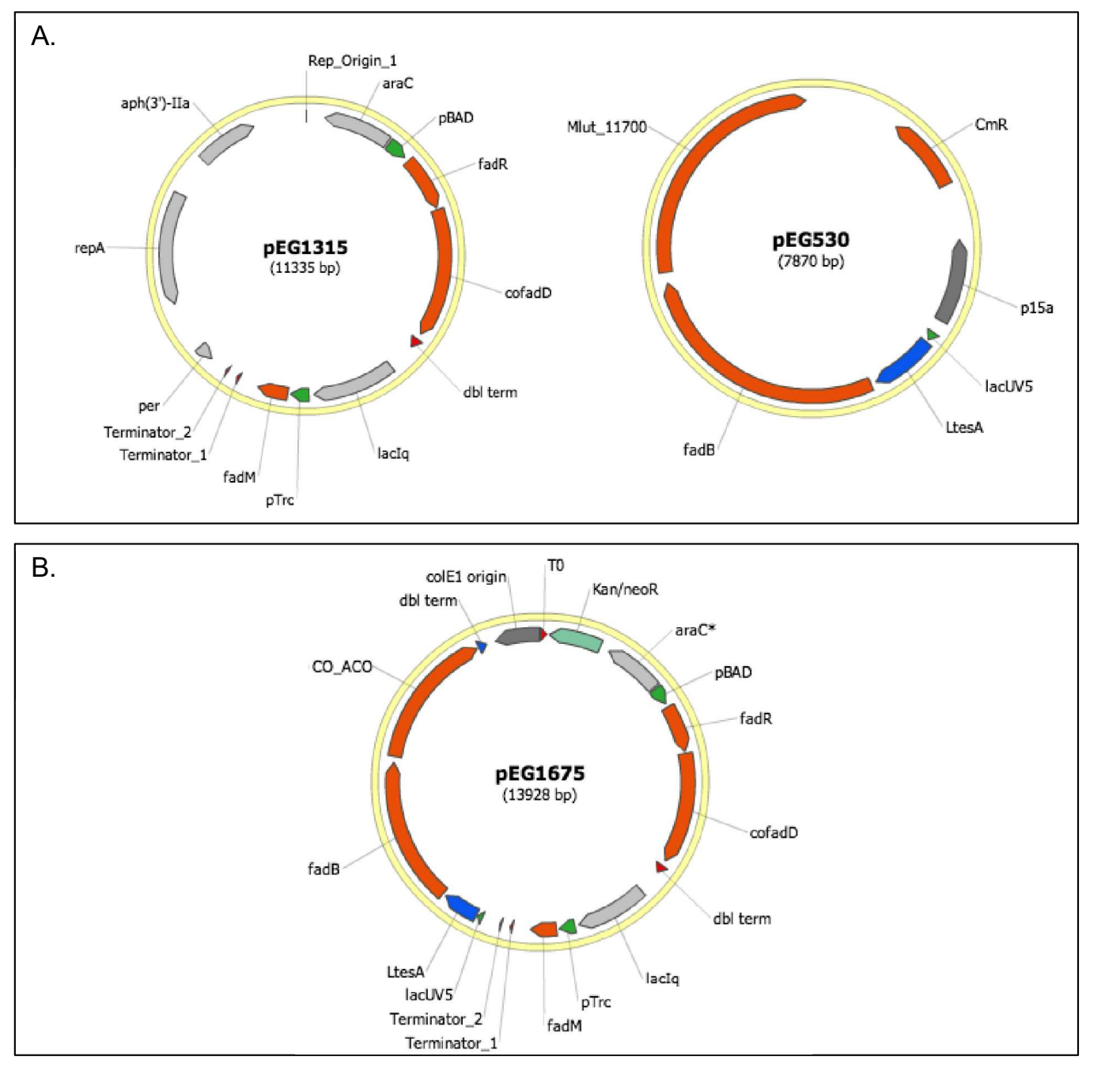

Figure 4

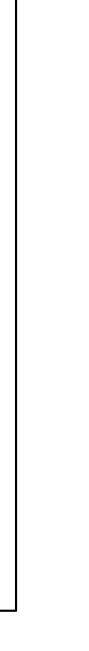

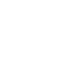



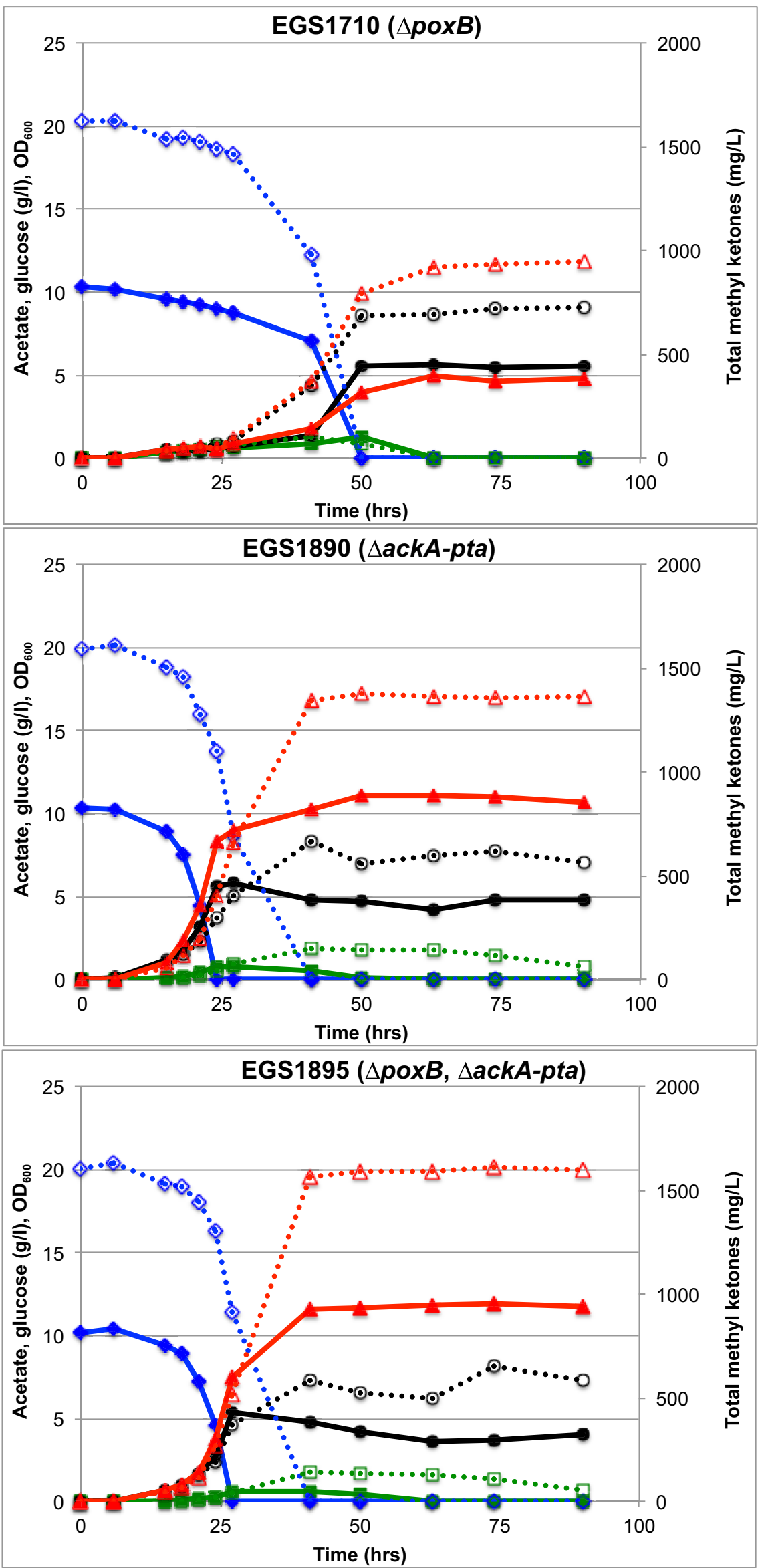


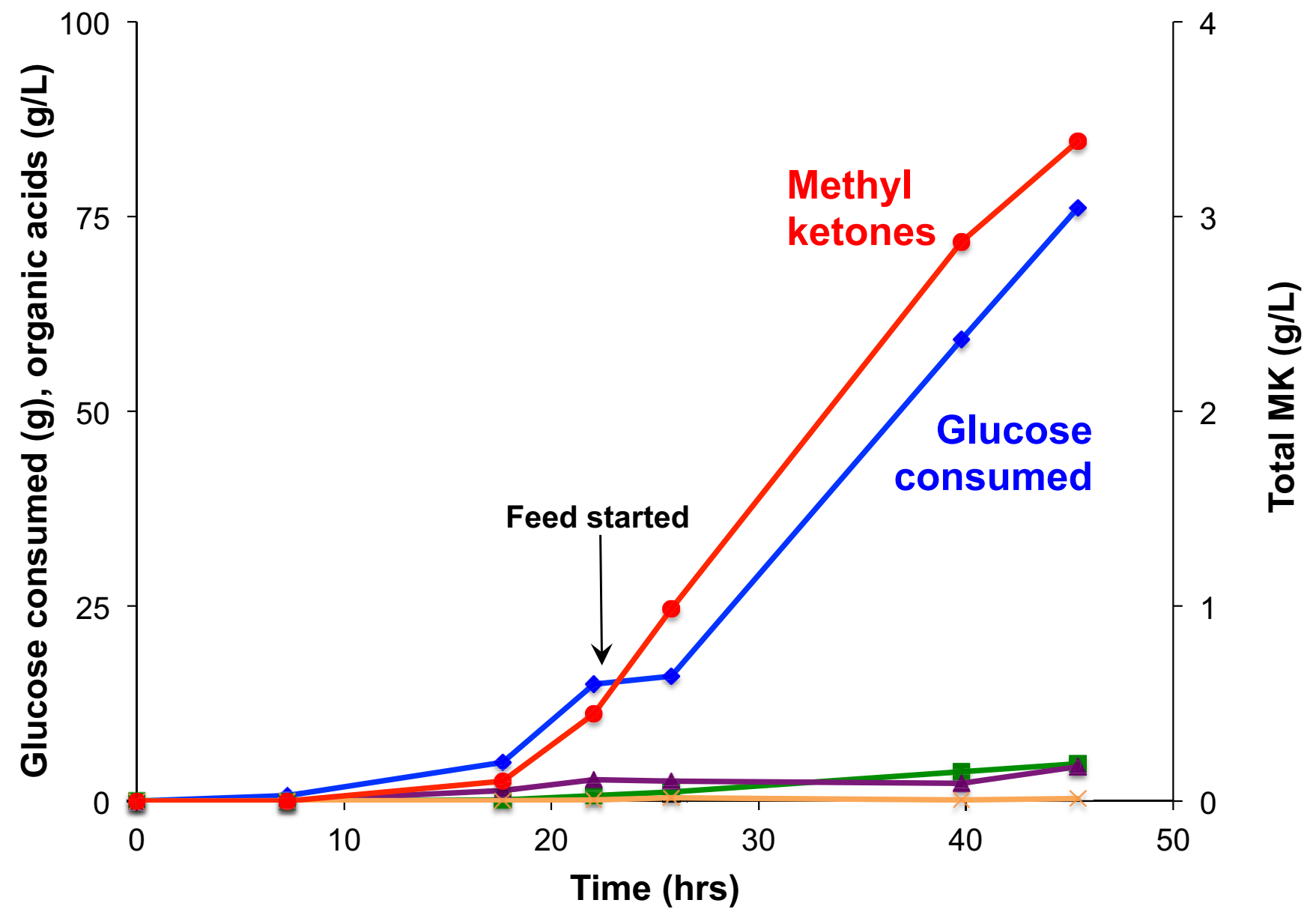

$\rightarrow$ Glucose consumed $\rightarrow$-Acetate $\rightarrow$ Pyruvate $\rightarrow$ Lactate $\rightarrow$-Total MK 